\title{
Hodgkin Lenfoma Olgularında Üç Farklı Radyoterapi Tekniği Kullanılarak Hedef Hacim ve Kritik Organ Dozlarının Retrospektif İncelenmesi
}

\author{
Hamit Başaran ${ }^{1 *}$, Gökçen İnan ${ }^{2}$ \\ ${ }^{1 *}$ Selçuk Üniversitesi, Tıp Fakültesi, Radyasyon Onkolojisi, Konya, Türkiye, (ORCID: 0000-0002-2122-8720), drhbasaran@gmail.com \\ ${ }^{2}$ Selçuk Üniversitesi, Tıp Fakültesi, Radyasyon Onkolojisi, Konya, Türkiye (ORCID: 0000-0003-2995-0256), gokceninan85@gmail.com
}

(İlk Geliş Tarihi 15 Ekim 2021 ve Kabul Tarihi 15 Aralık 2021)

(DOI: 10.31590/ejosat.1010145)

ATIF/REFERENCE: Başaran, H. \& İnan, G. (2021). Hodgkin Lenfoma Olgularında Üç Farklı Radyoterapi Tekniği Kullanılarak Hedef Hacim ve Kritik Organ Dozlarının Retrospektif İncelenmesi. Avrupa Bilim ve Teknoloji Dergisi, (31), 320-325.

$\ddot{O} \mathbf{z}$

$\mathrm{Bu}$ çalışmada bilateral boyun ve mediasten tutulumu olan Hodgkin Lenfoma (HL) olgularında alan içinde alan (Field in Field (FinF)), butterfly yoğunluk ayarlı radyoterapi (B-YART) ve rainbow yoğunluk ayarlı radyoterapi (R-YART) tekniklerinin dozimetrik olarak karşılaştırılması amaçlanmıştır. Oluşturulan tedavi planları planlanan hedef hacim (Planned Target Volume-(PTV)) dozları, konformite indeksi (CI), homojenite indeksi (HI) ve monitör ünit (MUs) açısından değerlendirildi. Ayrıca kritik organların (total akciğer, kalp, spinal kord, tiroid, larenks, özafagus, sağ parotis ve sol parotis) $\mathrm{D}_{\text {ort }}(\mathrm{Gy}), \mathrm{V}_{5}, \mathrm{~V}_{10}, \mathrm{~V}_{20}$ ve $\mathrm{V}_{30}$ dozları karşılaştırıldı. PTV için ortalama dozlar FinF tekniğinde $30.19 \pm 0.17$ Gy iken, B-YART ve R-YART tekniklerinde sirasıyla $30.74 \pm 0.19$ Gy ve $30.80 \pm 0.13^{\prime}$ dür. Bu üç teknik için PTV' nin aldığı ortalama dozlar benzerdir. Kritik organlardan bilateral akciğer ortalama dozlarının benzer olduğu görülmüştür. Fakat FinF planlarında $V_{5}$ gibi düşük doz alan hacim, B-YART ve R-YART planlarında ise $\mathrm{V}_{20}$ ve $\mathrm{V}_{30}$ gibi yüksek doz alan hacim anlamlı olarak düşük bulunmuştur $(p<0.05)$. Tiroid ve larenks için $D_{\text {ort }}$ ve $V_{30}$ değerleri B-YART ve R-YART planlarında anlamlı şekilde düşük olduğu görüldü ( $p$ değerleri sırası ile $0.001,0.003$ ). HL tedavisinde $V_{20}$ ve $V_{30}$ gibi yüksek doz alan hacimlerde B-YART ve R-YART tekniklerinin FinF tekniğine göre daha avantajlı olduğu görülmektedir. HL tedavilerinde kritik yapıların aldığı dozlara bakılarak kişiye uygun planlama tercih edilebilir.

Anahtar Kelimeler: Butterfly, Hodgkin Lenfoma, Rainbow, Yoğunluk Ayarlı Radyoterapi

\section{Evaluation of Target Volume and Critical Organ Doses in Hodgkin Lymphoma Cases Using Three Different Radiotherapy Techniques}

\begin{abstract}
In this study, it was aimed to compare the dosimetric methods of field-in-field (Field in Field (FinF)), butterfly intensity modulated radiotherapy (B-IMRT), and rainbow intensity modulated radiotherapy (R-IMRT) in Hodgkin Lymphoma (HL) cases. Plans were evaluated in terms of planned target volume (PTV) doses, conformity index (CI), homogeneity index (HI), and monitor unit (MUs). In addition, $\mathrm{D}_{\text {ort }}(\mathrm{Gy}), \mathrm{V}_{5}, \mathrm{~V}_{10}, \mathrm{~V}_{20}$ and $\mathrm{V}_{30}$ doses for total lung, heart, spinal cord, thyroid, larynx, esophagus, right parotid and left parotid were compared as critical organs. The mean doses for PTV were 30.19 \pm 0.17 Gy for the FinF technique, and $30.74 \pm 0.19$ Gy and 30.80 \pm 0.13 for the B-IMRT and R-IMRT techniques, respectively. The average doses received by PTV for the three techniques are similar. It was observed that the mean bilateral lung doses for the plans were similar, but it was found to be significantly lower in the low-dose volume FinF plans such as $\mathrm{V}_{5}$, and high-dose volumes such as $\mathrm{V}_{20}$ and $\mathrm{V}_{30}$ in the B-IMRT and R-IMRT plans (p<0.05). $\mathrm{D}_{\text {ort }}$ and $\mathrm{V}_{30}$ dose values for thyroid and larynx were found to be significantly lower in B-IMRT and R-IMRT plans ( $\mathrm{p}$ values 0.001 , 0.003, respectively). In the treatment of HL, B-IMRT and R-IMRT techniques seem to be more advantageous than FinF technique in high dose volumes such as $\mathrm{V}_{20}$ and $\mathrm{V}_{30}$. In HL treatments, individual planning can be preferred by looking at the doses taken by critical structures.
\end{abstract}

Keywords: Hodgkin's Lymphoma, Butterfly, Intensity-Modulated Radiotherapy, Rainbow

${ }^{1}$ Sorumlu Yazar: $\underline{\text { drhbasaran } @ \text { gmail.com }}$ 


\section{Giriş}

Kemoterapi ve radyoterapi tedavi bileşenleri erken evre hodgkin lenfoma (HL) hastalarında önemli bir rol oynamaktadır $(1,2)$. Bu nedenle kemoterapi ve radyoterapi, tek başına yada kombine olarak uygulanabilmektedir. $\mathrm{Bu}$ tedavi yöntemleri ile lenfomalarda, özellikle HL hastalarında kür oranı yüksektir. Ancak lenfoma tedavisinin amacı en az komplikasyon ile kür sağlamak olmalıdır. Hodgkin lenfomasından etkilenen hastalarda yüksek kür oranlarına ulaşmak, kemoterapi ve radyasyondan kaynaklanan toksisiteyi en aza indirme ihtiyacı ile etkili tedaviyi dengelemeye bağlıdır $(3,5)$.

Erken evre HL etkilenen hastaların genel prognozu mükemmeldir ve toplam 15 yıllık sağkalım oranı \%80'den fazladır (6). Radyoterapi alan boyutunda ve dozunda azalmanın yanı sıra gelişmiş radyoterapi teknikleri, HL hastalarında normal doku maruziyetini daha da azaltabilir (7). Çoğu HL hastasının tedaviden sonra uzun süreli sağkalım elde etmesiyle, özellikle meme ve akciğer kanseri $(8,9)$ ve kardiyovasküler hastalık gibi ikinci maligniteler olmak üzere uzun vadeli komplikasyon riski çok önemlidir $(10,11)$. Son dönemlerde modern konformal radyoterapi teknikleriyle birlikte, modern ileri görüntüleme sistemleri ve görüntü füzyonu sayesinde tedavi algoritmaları oldukça gelişmiş ve işınlanan tedavi hacimleri azaltılarak, normal dokuların aldığı gereksiz dozlarda azalma sağlanmıştır. Böylece uzun vadeli komplikasyon riskini en aza indirerek oldukça kişiselleştirilmiş tedaviler planlanabilmektedir.

$\mathrm{Bu}$ çalışmada erken evre HL tanılı bilateral boyun ve mediasten yerleşimli olgularda, field-in-field (Field in Field (FinF)) ve iki farklı yoğunluk ayarlı radyoterapi (YART) tekniklerinin (butterly- yoğunluk ayarlı radyoterapi (B-YART ve rainbow yoğunluk ayarlı radyoterapi (R-YART)) dozimetrik olarak karşılaştırılması amaçlanmıştır.

\section{Materyal ve Metot}

\subsection{Hasta Seçimi ve Görüntüleme}

Bu çalışmaya Selçuk Üniversitesi, Tıp Fakültesi, Radyasyon Onkolojisi Anabilim Dalı'nda bilateral boyun ve mediastinal tutulumu olan HL tanısı ile radyoterapi almış olan 10 hasta seçildi. Yapılan bu çalışma için Selçuk Üniversitesi, Tıp Fakültesi, Etik Kurulu'ndan 28 Eylül 2021 tarihli ve 2021/428 nolu karar ile izin alındı. Hastalar sırtüstü pozisyonda baş ve boyun termoplastik maske ile sabitlendi ve $3 \mathrm{~mm}$ 'lik kesit aralığ ile bilgisayarlı tomografi (BT) görüntüleri elde edildi. BT görüntüleri tedavi planlama sistemine (TPS) (Eclipse, sürüm 15.1; Varian) aktarıldı.

\subsection{Hedef Hacim ve Kritik Organlar}

Hastaların kemoterapi öncesi ve sonrasi F-18 florodeoksiglukoz-pozitron emisyon tomografisi bilgisayarlı tomografi (FDG-PET BT) taramaları planlama tomografisi ile füzyon edildi ve Görünür Hedef Hacim (GTV) olarak kaydedildi. Klinik Hedef Hacim (CTV) ve Risk Altındaki Organlar (OAR) aynı radyasyon onkoloğu tarafindan şekillendirildi. CTV için üst sınırı C5-C6 aralıktan başlayarak bilateral servikal, supraklaviküler ve infraklaviküler lenf nodları dahil edildi. Alt kisımda ise medyastinel ve bilateral hiler lenfnodları alana dahil edildi. Tüm hastalar için akciğerler, kalp spinal kord, tiroid, larenks, özafagus ve parotid bezleri OAR olarak BT veri setlerinde tanımlandı. EORTC-GELA (Groupe d'Études des Lymphomes de l'Adulte) H8 çalışma kılavuzlarına göre (12), CTV kemoterapi öncesi CTV (kemoterapi öncesi BT ve PET-CT taramaları temelinde şekillendirilmiş) olarak tanımlandı. Fakat kemoterapi sonrası anatomik sınırlara göre modifiye edildi. $\mathrm{Bu}$ klavuzda önerildiği gibi, büyük kan damarları ve kalp, mümkün olduğu kadar CTV'ye dahil edilmedi. Planlama Hedef Hacmi (PTV) oluşturmak için CTV' ye 8 mm'lik bir marj eklendi (mediyastinal lenf nodlarinin internal motion telafi etmek için $5 \mathrm{~mm}$ ve set-up hatası için 3 $\mathrm{mm})(13)$.

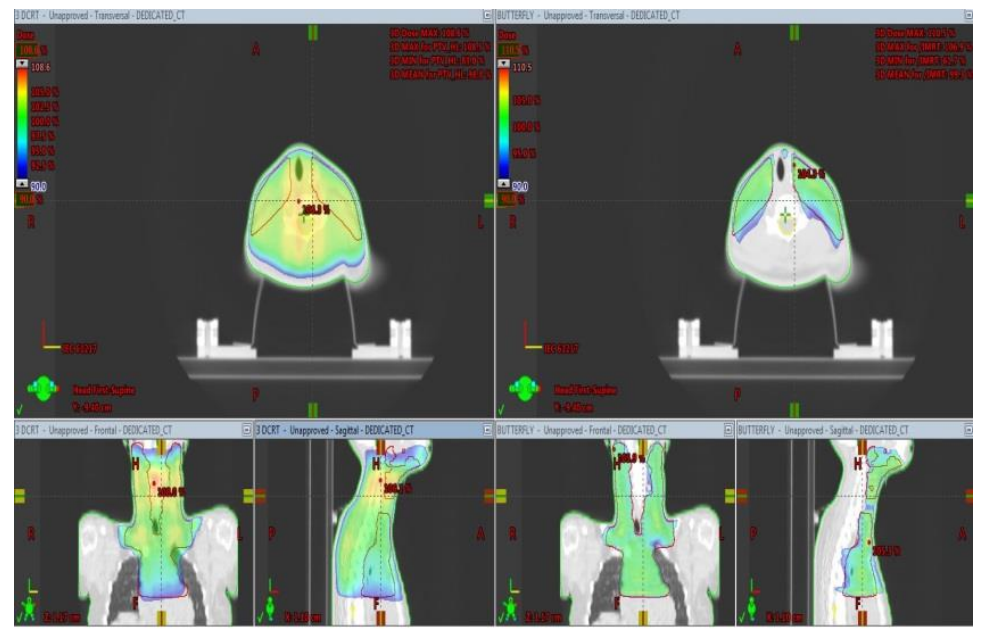

Şekil 1. FinF ve B-YART planlarının doz karşılaştırması

\subsection{Radyoterapi Planlama}

Radyoterapi planlaması için Varian marka (Varian Medical Systems, Palo Alto, CA) tedavi cihazı ve tedavi planlama sistemi (TPS) (Eclipse ver. 15.1) kullanılarak üç farklı teknikte (FinF, BYART ve R-YART) tedavi planları yapıldı. Radyoterapi dozu günük 1.8 Gy ile toplamda 30.6 Gy olacak şekilde planlandı. FinF tedavi tekniği için karşılıklı iki alan AP/PA (anteroposterior ve postero-anterior) kullanıldı. FinF planları ön alan enerjisi $6 \mathrm{MV}$, arka alan enerjisi $18 \mathrm{MV}$ olarak seçildi. Daha sonra her bir ışın ana alan harici 2 alt alan (segment) eklenerek PTV'de ki doz fazlalılığı azaltılmaya çalışıldı. Bu işlem sırasında ana alan ve alt alanlara uygun ağırlıkların verilmesine önem verildi. Çalışmada YART planları ise tersten planlama (inverse planing) yöntemiyle 5 alandan oluşan B-YART ve RYART teknikleri kullanılarak hazırlandı. B-YART tekniği 1 ön, 2 ön oblik ve 2 arka oblik, R-YART tekniği ise 1 ön ve 4 ön oblik ışın alanı kullanılacak şekilde planlandı. Her iki YART planlarında da $6 \mathrm{MV}$ enerji kullanıldı. Tüm tedavi planları Anizotropik Analitik Algoritma (AAA) ile hesaplatıldı. 


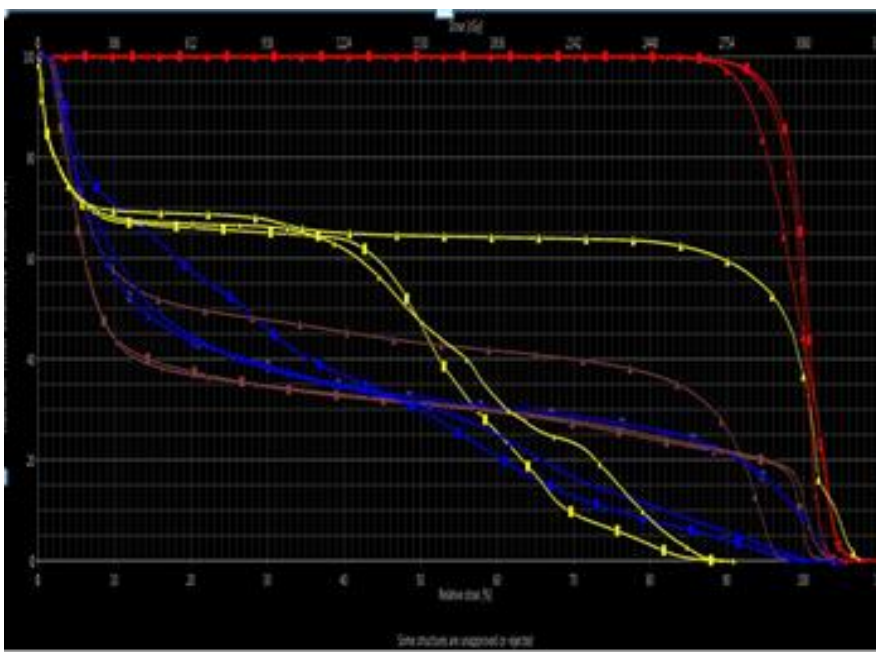

Şekil 2. FinF, B-YART ve R-YART teknikleri ile tedavi planı hazırlanmış olan bir hastanın doz volüm histogramı karşılaştırması ( $\mathbf{\Delta}$ : FinF tekniği •B-YART tekniği, $\mathbf{~}:$ R-YART tekniği, tekniği kırmızı: PTV, mavi: akciğer, kahverengi: kalp, sar1: spinal kord)

\subsection{Plan Değerlendirme ve İstatiksel Analiz}

Çalışmada yapılan tedavi planlarının hedef ve kritik organ dozlarının değerlendirilmesinde doz volüm histogramlarından (DVH) yararlanıldı. FinF ve iki ayrı YART planlarından elde edilen DVH'ler karşılaştırılarak PTV, total akciğer, kalp, spinal kord, tiroid, sağ parotis, sol parotisin aldığ 1 dozlar değerlendirildi. Homojenite indeksi (HI) ICRU tarafindan yayınlanan Rapor 83'e göre tanımlandı (14).

Tablo 1. Planlanan hedef hacimdeki dozimetrik değerler

$$
\mathrm{HI}=\frac{\left(D_{2 \%}-D_{98 \%}\right)}{D_{50 \%}}
$$

Bu formülde $\mathrm{D}_{2 \%}, \mathrm{D}_{50 \%}$ ve $\mathrm{D}_{98 \%}$, sirsiyla hedef hacmin $\% 2, \% 50$ ve $\% 98$ 'inin aldığı doz olarak tanımlanmaktadır. HI değerinin 0 olması hedef hacmin homojen doz dağılımına sahip olduğunu göstermektedir. Konformite İndeksi, tedavi edilen hacmin (TV), planlanan hedef hacime (PTV) oranıdır. CI=TV/ PTV olarak tanımlanır. CI 1'e eşit olduğu durumlar ise ideal doz dağılımını ifade etmektedir. CI 1'den büyük ise ışınlanan hacim hedef hacimden büyük, 1' den küçük ise hedef hacim kısmi olarak ışınlanmaktadır (15). Bu değer TPS ile otomatik olarak elde edildi. DVH' ları kullanılarak PTV'ye ait $\mathrm{D}_{\text {mak }}(\mathrm{Gy}), \mathrm{D}_{\text {ort }}$ (Gy) (hedef hacimde maksimum ve ortalama dozlar), $\mathrm{D}_{98}, \mathrm{D}_{95}$, $\mathrm{D}_{50}$, ve $\mathrm{D}_{2}$ verileri karşılaştırıldı. Total akciğer, kalp, spinal kord, tiroid, larenks, özafagus, sağ parotis ve sol parotis için $\mathrm{D}_{\text {ort }}(\mathrm{Gy})$, $\mathrm{V}_{5}, \mathrm{~V}_{10}, \mathrm{~V}_{20}$ ve $\mathrm{V}_{30}$ dozları karşılaştırıldı. Ayrıca planların MU' leri değerlendirildi.

\section{5. İstatistiksel Analiz}

Statistical Package for Social Sciences (SPSS) 25.1 versiyonu kullanılarak istatistiksel analizi yapıldı. İstatistiksel analizde iki grup arasındaki fark için Paired samples T-test kullanıldı ve $\mathrm{p}<0.05$ değeri anlamlı kabul edildi.

\section{Araştırma Sonuçları ve Tartışma}

\subsection{Bulgular}

FinF, B-YART ve R-YART tedavi planlamalarından elde edilen PTV'ye ait doz değerleri, HI, CI ve MU değerleri Tablo 1.' de verildi. PTV' ye ait tanımlanan doz ile kapsaması açısından ( $D_{\text {mak }}, D_{\text {ort }}$ ) üç planda da anlamlı fark bulunmadı. HI' nın en ideal değeri " 0 " olduğundan en homojen doz dağılımının oluştuğu planlar B-YART ve R-YART tekniklerinde anlamlı bulundu ( $p=0.007, p=0.009)$. CI' nın en ideal değeri " 1 " olduğundan en konformal teknik aynı şekilde B-YART ve RYART tekniklerinde anlamlı değer elde edildi $(p=0.000)$. FinF, B-YART ve R-YART teknikleri için ortalama MU sayıları sirasiyla $180 \pm 4,1258 \pm 108$ ve $1067 \pm 65$ olarak bulundu. FinF tekniği için gerekli olan MU değerinin anlamlı olarak daha düşük olduğu gözlendi $(\mathrm{p}=0.000)$.

Tablo 2.'de kritik organlar için üç teknik arasındaki dozimetrik değerlerin karşılaştırılması verildi. Üç farklı planlama için bilateral akciğer ortalama dozlarının benzer olduğu görüldü. $V_{5}$ gibi düşük doz alan hacim 3 DCRT planlarında, $V_{20}$ ve $V_{30}$ gibi yüksek doz alan hacim B-YART ve R-YART planlarında anlamlı olarak düşük bulundu $(\mathrm{p}<0.05)$. Kalbin $\mathrm{D}_{\text {ort }}, \mathrm{V}_{10}$ ve $\mathrm{V}_{20}$ dozları B-YART ve R-YART tekniklerinde, FinF tekniğine göre daha düşüktü ve istatistiksel olarak anlamlıydı $(\mathrm{p}<0.05)$. Spinal kord $\mathrm{D}_{\text {ort, }} \mathrm{V}_{10}$ ve $\mathrm{V}_{20}$ dozları FinF planlarında, B-YART ve R-YART planlarında göre anlamlı olarak yüksek bulundu ( $p$ değerleri sırası ile $0.016,0.017,0.030$ ). Özafagus $V_{20}$ ve $V_{30}$ gibi yüksek doz alan hacim B-YART ve R-YART planlarında anlamlı olarak düşük olduğu bulundu. Tiroid ve larenks için $D_{\text {ort }}$ ve $V_{30}$ doz değerleri B-YART ve R-YART planlarında anlamlı şekilde düşük olduğu görüldü (p değerleri sırasi ile $0.001,0.003$ ). Sağ ve sol parotis $\mathrm{D}_{\text {ort }}$ ve $\mathrm{V}_{20}$ değerleri $\mathrm{B}$-YART tekniğinde, R-YART ve FinF tekniklerine göre anlamlı derece düşük bulundu ve istatistiksel olarak anlamlı kabul edildi $(\mathrm{p}=0.001)$. 
Tablo 1. Planlanan hedef hacimdeki dozimetrik değerler

\begin{tabular}{|c|c|c|c|c|c|c|}
\hline \multirow[t]{2}{*}{ Parametre } & \multirow{2}{*}{$\begin{array}{l}\text { FinF } \\
(\text { Ort } \pm \text { SS) }\end{array}$} & \multirow{2}{*}{$\begin{array}{l}\text { (B) } \\
\text { BUTTERFLY } \\
\text { YART } \\
(\text { Ort } \pm \text { SS })\end{array}$} & \multirow{2}{*}{$\begin{array}{l}\text { (C) } \\
\text { RAINBOWYA } \\
\text { RT } \\
(\text { Ort } \pm \text { SS) }\end{array}$} & \multicolumn{3}{|c|}{ p value } \\
\hline & & & & A vs. B & A vs. C & B vs. C \\
\hline PTV D98 & $27.67 \pm 0.45$ & $28.50 \pm 0.33$ & $28.76 \pm 0.49$ & 0.001 & 0.005 & 0.166 \\
\hline PTV D95 & $28.01 \pm 0.58$ & $29.18 \pm 0.35$ & $29.44 \pm 0.41$ & 0.004 & 0.005 & 0.062 \\
\hline PTV D50 & $30.09 \pm 0.27$ & $30.89 \pm 0.17$ & $30.90 \pm 0.13$ & 0.147 & 0.234 & 0.881 \\
\hline PTV D2 & $32.49 \pm 0.25$ & $32.28 \pm 0.40$ & $32.34 \pm 0.20$ & 0.126 & 0.158 & 0.639 \\
\hline PTV mak & $33.16 \pm 0.22$ & $32.57 \pm 0.34$ & $32.70 \pm 0.60$ & 0.154 & 0.142 & 0.583 \\
\hline PTV ort & $30.19 \pm 0.17$ & $30.74 \pm 0.19$ & $30.80 \pm 0.13$ & 0.128 & 0.215 & 0.379 \\
\hline CI & $1.65 \pm 0.10$ & $0.71 \pm 0.10$ & $0.76 \pm 0.08$ & 0.000 & 0.000 & 0.097 \\
\hline HI & $0.16 \pm 0.026$ & $0.11 \pm 0.007$ & $0.11 \pm 0.01$ & 0.007 & 0.009 & 0.224 \\
\hline MU & $180 \pm 4$ & $1258 \pm 108$ & $1067 \pm 65$ & 0.000 & 0.000 & 0.010 \\
\hline
\end{tabular}

Tablo 2. Risk altındaki organlarda dozimetrik değerler

\begin{tabular}{|c|c|c|c|c|c|c|c|}
\hline \multirow[t]{2}{*}{ Organ } & \multirow{2}{*}{$\begin{array}{c}\text { Parame } \\
\text { tre }\end{array}$} & \multirow{2}{*}{$\begin{array}{c}\text { (A) } \\
\text { FinF } \\
(\text { Ort } \pm \text { SS })\end{array}$} & \multirow{2}{*}{$\begin{array}{c}\text { B) } \\
\text { BUTTERFLY } \\
\text { YART } \\
(\text { Ort } \pm \text { SS })\end{array}$} & \multirow{2}{*}{$\begin{array}{c}(\mathrm{C}) \\
\text { RAINBOW } \\
\text { YART } \\
(\text { Ort } \pm \text { SS })\end{array}$} & \multicolumn{3}{|c|}{ p value } \\
\hline & & & & & A vs. B & A vs. C & B vs. C \\
\hline \multirow[t]{5}{*}{ Total Akciğer } & Dort & $9.05 \pm 1.69$ & $8.26 \pm 1.86$ & $8.82 \pm 2.12$ & 0.053 & 0.571 & 0.425 \\
\hline & $V_{5}$ & $38.52 \pm .7 .98$ & $45.14 \pm 11.90$ & $57.52 \pm 15.30$ & 0.102 & 0.006 & 0.000 \\
\hline & $V_{10}$ & $30.11 \pm 5.74$ & $32.26 \pm 6.69$ & $38.41 \pm 9.87$ & 0.147 & 0.016 & 0.019 \\
\hline & $\mathbf{V}_{20}$ & $22.58 \pm 4.90$ & $13.41 \pm 4.34$ & $10.52 \pm 4.13$ & 0.000 & 0.000 & 0.001 \\
\hline & $\mathbf{V}_{30}$ & $5.99 \pm 4.31$ & $1.16 \pm 1.43$ & $0.59 \pm 0.90$ & 0.033 & 0.031 & 0.110 \\
\hline \multirow[t]{5}{*}{ Kalp } & Dort & $7.62 \pm 5.14$ & $6.07 \pm 4.10$ & $5.81 \pm 4.37$ & 0.016 & 0.043 & 0.615 \\
\hline & $V_{5}$ & $31.91 \pm 21.992$ & $26.42 \pm 19.56$ & $25.28 \pm 22.54$ & 0.100 & 0.292 & 0.753 \\
\hline & $V_{10}$ & $7.44 \pm 20.13$ & $19.80 \pm 14.94$ & $18.71 \pm 16.20$ & 0.017 & 0.024 & 0.699 \\
\hline & $V_{20}$ & $19.75 \pm 16.55$ & $12.37 \pm 11.04$ & $12.07 \pm 11.17$ & 0.030 & 0.024 & 0.723 \\
\hline & $\mathbf{V}_{30}$ & $0.00 \pm 0.00$ & $5.45 \pm 7.27$ & $5.69 \pm 6.51$ & 0.126 & 0.085 & 0.786 \\
\hline \multirow[t]{5}{*}{ Spinal Kord } & Dort & $21.26 \pm 2.36$ & $13.20 \pm 1.24$ & $11.01 \pm 1.49$ & 0.000 & 0.000 & 0.002 \\
\hline & $V_{5}$ & $72.37 \pm 7.80$ & $71.74 \pm 8.23$ & $70.19 \pm 10.17$ & 0.094 & 0.077 & 0.140 \\
\hline & $V_{10}$ & $69.74 \pm 7.76$ & $68.80 \pm 7.75$ & $60.97 \pm 11.31$ & 0.309 & 0.029 & 0.031 \\
\hline & $\mathbf{V}_{20}$ & $67.00 \pm 8.54$ & $24.87 \pm 5.28$ & $8.19 \pm 7.90$ & 0.000 & 0.000 & 0.003 \\
\hline & $\mathbf{V}_{30}$ & $46.53 \pm 13.08$ & $0.00 \pm 0.00$ & $0.03 \pm 0.09$ & 0.000 & 0.000 & 0.363 \\
\hline \multirow[t]{5}{*}{ Tiroid } & Dort & $31.28 \pm 0.60$ & $27.25 \pm 2.02$ & $28.25 \pm 1.75$ & 0.001 & 0.003 & 0.156 \\
\hline & $V_{5}$ & $81.43 \pm 36.02$ & $100 \pm 0.00$ & $100 \pm 0.00$ & 0.262 & 0.262 & - \\
\hline & $V_{10}$ & $96.02 \pm 9.74$ & $84.45 \pm 36.48$ & $100 \pm 0.00$ & 0.507 & 0.363 & 0.344 \\
\hline & $\mathbf{V}_{20}$ & $95.69 \pm 10.55$ & $88.10 \pm 11.37$ & $96.39 \pm 4.41$ & 0.370 & 0.899 & 0.121 \\
\hline & $V_{30}$ & $91.10 \pm 14.44$ & $44.98 \pm 10.74$ & $45.26 \pm 15.63$ & 0.003 & 0.003 & 0.944 \\
\hline \multirow[t]{5}{*}{ Larenks } & Dort & $31.21 \pm 0.42$ & $21.34 \pm 6.40$ & $24.47 \pm 3.73$ & 0.014 & 0.008 & 0.051 \\
\hline & $V_{5}$ & $100 \pm 0.00$ & $100 \pm 0.00$ & $100 \pm 0.00$ & - & - & - \\
\hline & $V_{10}$ & $100 \pm 0.00$ & $85.99 \pm 16.30$ & $100 \pm 0.00$ & 0.089 & - & 0.082 \\
\hline & $\mathbf{V}_{20}$ & $100 \pm 0.00$ & $55.39 \pm 32.24$ & $78.64 \pm 19.27$ & 0.019 & 0.042 & 0.023 \\
\hline & $\mathbf{V}_{30}$ & $93.61 \pm 11.02$ & $24.45 \pm 12.46$ & $24.51 \pm 12.21$ & 0.007 & 0.007 & 0.968 \\
\hline \multirow[t]{5}{*}{ Özafagus } & Dort & $22.41 \pm 4.49$ & $19.69 \pm 6.03$ & $20.63 \pm 5.50$ & 0.086 & 0.265 & 0.108 \\
\hline & $V_{5}$ & $78.42 \pm 17.49$ & $76.65 \pm 17.76$ & $77.34 \pm 17.43$ & 0.022 & 0.235 & 0.200 \\
\hline & $V_{10}$ & $77.39 \pm 18.20$ & $67.99 \pm 15.27$ & $74.11 \pm 16.71$ & 0.003 & 0.124 & 0.027 \\
\hline & $\mathbf{V}_{20}$ & $73.89 \pm 18.52$ & $55.79 \pm 13.25$ & $50.50 \pm 20.53$ & 0.017 & 0.058 & 0.542 \\
\hline & $\mathbf{V}_{30}$ & $32.92 \pm 13.13$ & $12.89 \pm 6.18$ & $15.97 \pm 13.96$ & 0.043 & 0.034 & 0.514 \\
\hline \multirow[t]{5}{*}{ Sağ Parotis } & Dort & $19.34 \pm 6.14$ & $15.64 \pm 6.20$ & $18.01 \pm 6.63$ & 0.000 & 0.112 & 0.015 \\
\hline & $V_{5}$ & $81.29 \pm 19.63$ & $74.22 \pm 23.47$ & $82.87 \pm 20.22$ & 0.065 & 0.680 & 0.151 \\
\hline & $V_{10}$ & $72.58 \pm 24.70$ & $62.27 \pm 26.48$ & $72.13 \pm 25.42$ & 0.015 & 0.884 & 0.011 \\
\hline & $\mathrm{V}_{20}$ & $55.06 \pm 25.03$ & $37.62 \pm 22.48$ & $51.01 \pm 29.90$ & 0.001 & 0.349 & 0.069 \\
\hline & $V_{30}$ & $9.81 \pm 11.22$ & $12.37 \pm 13.71$ & $11.59 \pm 12.04$ & 0.760 & 0.824 & 0.630 \\
\hline
\end{tabular}




$\begin{array}{ccc}\text { Sol Parotis } & \text { Dort } & 21.06 \pm 3.79 \\ & \mathbf{V}_{\mathbf{5}} & 72.14 \pm 32.38 \\ \mathbf{V}_{\mathbf{1 0}} & 77.03 \pm 14.42 \\ \mathbf{V}_{\mathbf{2 0}} & 61.28 \pm 16.84 \\ & \mathbf{V}_{\mathbf{3 0}} & 19.93 \pm 10.92\end{array}$

\subsection{Tartışma}

Lenfoma tedavisinden elde edilen olumlu sonuçlar, tedavi morbiditesini en aza indirmeyi amaçlayan tedavi tekniklerinin geliştirilmesine yön vermektedir. Modern tedavi protokolleri, tedaviden sonra geç toksisiteye neden olduğu bilinen kemoterapi ve radyasyon maruziyetini en aza indirmek için özel olarak tasarlanmaktadır (15). Mediasten tutulumu olan HL hastalara radyasyon verilmesi hedefin akciğerler, meme, yemek borusu, kalp ve alt yapılar gibi kritik yapılara yakınlığı nedeniyle özellikle zordur. YART tedavisinin mediasten tutulumu olan lenfomada kullanımı birçok çalışmada araştırılmıştır $(7,16)$. YART tedavisinin akciğer ve meme (kadınlarda) gibi düşük dozlara maruz kalan doku hacmini arttırırken doz uyumunu da iyileştirdiği görülmektedir. YART tedavisinin AP-PA ile elde edilemeyen bir diğer avantajı, zor veya karmaşık anatomik konumlarda dozu uyarlama yeteneğidir. Örneğin, kalbin să̆ tarafındaki mediastinal düğümlerin ortak tutulumu geleneksel radyasyon planlaması için zordur, çünkü AP-PA 1şınları kaçınılmaz olarak göğüslerden girerken YART tekniği bu sorunu önlemek için ışın açısını değiştirme esnekliği sunmaktadır.

YART tedavisi her hasta için en iyi teknik olmayabilir; örneğin, hedef hacim bilateral aksilla içerdiğinde YART ile kabul edilebilir düşük akciğer dozları elde etmek daha zordur ve bu gibi durumlarda geleneksel AP-PA teknikleri daha iyi sonuç verebilir. Ayrıca YART tedavisinin dezavantajlarından biri de, kritik noktalara verilen düşük integral dozlardır.

Bazı radyasyon onkologları, normal yapıların yüksek doza maruz kalması ve meme gibi büyük hacimli normal dokulara düşük radyasyon dozu verilmesi endişeleri nedeniyle HL kadın hastalarda YART kullanma konusunda isteksizdir. Meme kanseri riski açıkça doza bağımlı olmasına rağmen, düşük doz radyasyon ve karsinojenez arasındaki ilişki daha spekülatiftir $(17,18)$. Weber ve ark. YART tekniğini geleneksel tekniklerle karşılaştırmak için doğrusal olmayan bir doz risk modeli kullanırken, YART'ın diğer konformal tekniklerle karşılaștırıldığında radyasyona bağlı kanserlerin tahmini riskini artırabileceği sonucuna varmışlardır (19).

Yapmış olduğumuz bu çalışma HL tanılı hastalarda özel bir 1şın düzenlemesi ile YART tedavilerinin geleneksel AP-PA tekniğine kıyasla normal yapıların yüksek radyasyon dozlarına maruz kalmasını etkili bir şekilde azaltabileceğini göstermektedir.

Goodman ve ark. HL ve nonhodgking lenfoma tanılı 16 hasta için YART, konvansiyonel paralel-karşıt (AP-PA) ve üç boyutlu konformal (3D-CRT) planlarını, 18 ila 45 Gy (medyan, 36 Gy) arasında değişen dozlar vermek üzere 6-MV X-1şınları kullanılarak tasarlamışlar ve planları doz-hacim parametreleri açısından karşılaştırmışlardır (16). Tüm hastalarda YART planlarını AP-PA ile karşılaştırdıklarında, YART tekniğinin ortalama akciğer dozunun, yaklaşık \%12 oranında azalttığını, ayrıca planlama hedef hacim kapsamı açısından daha iyi olduğu sonucuna varmışlardır. Yapmış oldukları çalışmalarında büyük

$\begin{array}{cccc}18.07 \pm 5.54 & \mathbf{0 . 0 0 1} & \mathbf{0 . 0 3 8} & 0.065 \\ 83.63 \pm 19.13 & 0.682 & 0.507 & 0.021 \\ 75.76 \pm 24.99 & 0.066 & 0.843 & 0.369 \\ 46.36 \pm 22.37 & \mathbf{0 . 0 0 1} & \mathbf{0 . 0 3 4} & 0.079 \\ 9.09 \pm 7.68 & 0.279 & 0.151 & 0.132\end{array}$

tedavi hacimlerinin RT' si için YART tekniğinin uygun olduğunu ancak doz uygulamasındaki iyileştirmelerin uzun vadeli morbiditeyi ve hastalık kontrolünü etkileyip etkilemediğini belirlemek için ek takibin gerekli olduğununu belirtmişlerdir. Yapmış olduğumuz çalışmada bu çalışmay ile benzer sonuçlar göstermektedir.

Akkaş ve ark. yapmış oldukları çalışmalarında erken evre HL tanılı bilateral boyun ve mediasten yerleşimli hastalara 3D CRT ile volumetric modulated arc therapy (VMAT) ve butterflyVMAT tekniklerini dozimetrik olarak karşılaştırmışlardır (20). Karşılaştırmalar sonucunda bilateral akciğer için ortalama dozlarda fark olmadığını ancak akciğer $\mathrm{V}_{5}$ değerlerinin ikili karşılaştırmalarda butterfly-VMAT planlamada, VMAT planlamaya göre istatistiksel olarak anlamlı düşük olduğu sonucuna varmışlardır (p: 0,012). Ayrıca sağ ve sol parotis ortalamaları, $\mathrm{V}_{5}, \mathrm{~V}_{20}, \mathrm{~V}_{25}$ ve $\mathrm{V}_{30}$ değerleri butterfly-VMAT tekniğinde diğer tekniklere göre anlamlı olarak daha düşük olduğunu belirtmişlerdir. Yapmış olduğumuz çalışmada da sağ ve sol parotis $\mathrm{D}_{\text {ort }}$ ve $\mathrm{V}_{20}$ değerleri B-YART tekniğinde, R-YART ve FinF tekniklerine göre anlamlı derece düşük bulundu ve istatistiksel olarak anlamlı kabul edildi $(\mathrm{p}=0.001)$.

Fiandra ve ark. yapmış oldukları çalışmalarında erken evre mediastinal HL'den etkilenen ve kemoterapi sonrası 30 Gy INRT (Involved Nodes Radiation Therapy) ile tedavi edilen toplam 10 hasta seçmişlerdir (21). Beş farklı tedavi tekniğini karşılaştırmışlardır (3D-CRT, VMAT (tek ark), B-VMAT ("butterfly", çoklu ark), Helikal Tomoterapi (HT) ve Tomoterapi). Tiroid bezi ve kalp/koroner için, HT, VMAT ve BVMAT teknikleri, 3D-CRT ve TD'ye kıyasla hem $\mathrm{D}_{\text {mean }}$ hem de orta-yüksek doz alan hacimler açısından daha iyi olduğunu belirtmişlerdir. B-VMAT planlarının hedef etrafında daha yüksek konformasyon ve daha düşük dozlara maruz kalan kritik organlar arasında en dengeli planlama oldukları sonucuna ulaşmışlardır.

Lloyd ve ark.11 hasta için akciğer, kalp ve memenin aldığ 1 dozlara odaklanarak AP/PA, YART ve Volumetrik Ark (VA) kullanarak mediastinal HL planlarını karşılaştırmışlardır (22). Akciğer $\mathrm{V}_{5}$ değerlerinin AP/PA planlarında YART ve VA planlarına kıyasla yaklaşık \%30 daha düşük olduğunu, ayrıca $\mathrm{V}_{20}$ değerinin ise AP/PA planlarında YART veya VA'dan \%25 daha fazla olduğunu bulmuşlardır. Yapmış olduğumuz çalışmada da benzer şekilde $V_{5}$ gibi düşük doz alan hacim 3D CRT planlarında, $V_{20}$ ve $V_{30}$ gibi yüksek doz alan hacim B-YART ve R-YART planlarında anlamlı olarak düşük bulundu.

\section{Sonuç}

Bilateral boyun ve mediasten tutulumu olan HL hastalar için akut ve geç dönem yan etkilerini değerlendirmede doz volüm sınırlamaları dikkate alınmalıdır. Bu çalışmada YART teknikleri FinF tekniğine kıyasla üstün hedef kapsamı ve kritik organ koruması sağlamıştır. Tedavi planlarında optimum 1şın seçimi her bir hasta için risk altındaki organların anatomisine ve öncelik sırasına göre bireysel olarak seçilmelidir. 


\section{Kaynakça}

Engert, A., Plutschow, A. \& Eich HT et al. (2010). Reduced treatment intensity in patients with early-stage Hodgkin's lymphoma. N Engl J Med, 363: 640-52.

Eich, S., Diehl, V. \& Gorgen, H. et al. (2010). Intensified chemotherapy and dose-reduced involved-field radiotherapy in patients with early unfavorable Hodgkin's lymphoma: Final analysis of the German Hodgkin Study Group HD11 trial. J Clin Oncol, 28: 4184- 4207.

Girinsky, T., Van der Maazen R., \& Specht L. et al. (2006). Involved-node radiotherapy (INRT) in patients with early Hodgkin lymphoma: concepts and guidelines. Radiother Oncol, 79: 270-277.

Bonadonna, G., Bonfante V. \& Viviani, S. et al. (2004). ABVD plus subtotal nodal versus involved-field radiotherapy in early-stage Hodgkin's disease: long-term results. J Clin Oncol, 22:2835-2841.

Noordijk, EM., Carde, P. \& Dupouy, N. et al. (2006). Combinedmodality therapy for clinical stage I or II Hodgkin's lymphoma: long-term results of the European organisation for research and treatment of cancer $\mathrm{H} 7$ randomized controlled trials. J Clin Oncol, 24:3128-3135.

Fiandra, C., Filippi, A.R. \& Catuzzo, P. et al. (2012). Different IMRT solutions vs. 3D-Conformal Radiotherapy in early stage Hodgkin's lymphoma: dosimetric comparison and clinical considerations. Radiation Oncology, 7:186.

Maraldo, M.V. \& Specht, L. (2014 A decade of comparative dose planning studies for early-stage Hodgkin lymphoma: What can we learn? Int J Radiat Oncol Biol Phys 90: 112635 .

Hodgson, D.C., Gilbert, E.S. \& Dores, G.M. et al. (2007). Long term solid cancer risk among 5-year survivors of Hodgkin's lymphoma. J Clin Oncol, 25:1489-1497.

Ng, A.K., Bernardo, M.V. \& Weller, E. et al. (2002). Second malignancy after Hodgkin disease treated with radiation therapy with or without chemotherapy: Long term risks and risk factors. Blood, 100:1989-1996.

Swerdlow, A.J., Higgins, C.D. \& Smith, P. et al. (2007). Myocardial infarction mortality risk after treatment for Hodgkin disease: a collaborative British cohort study. J Natl cancer Inst, 99:206-214.

Moser, E.C., Noordijk, E.M. \& Van Leeuwen, F.E. et al. (2006). Long-term risk of cardiovascular disease after treatment for aggressive non-Hodgkin lymphoma. Blood, 107:2912-2919.

Ferme, C., Eghbali, H. \& Meerwaldt, J.H. et al. (2007). EORTCGELA H8 Trial. Chemotherapy plus involved-field radiation in early-stage Hodgkin's disease. $N$ Engl J Med, 8;357(19):1916-27.

Fiandra, C., Filippi, A.R. \& Catuzzo, P. et al. (2012). Different IMRT solutions vs. 3D-conformal radiotherapy in early stage Hodgkin's Lymphoma: dosimetric comparison and clinical considerations. Radiat Oncol, 2;7:186.

ICRU Report 83 Prescribing, recording, and Reporting Photon Beam Intensity Modulated Radiation Therapy (IMRT). J ICRU, 2010. 10:1 106.
Oeffinger, K.C., Mertens, A.C. \& Sklar, C.A. et al. (2006). Chronic health conditions in adult survivors of childhood cancer. New Engl J Med, 355:1572-1582.

Goodman, K.A., Toner, S. Hunt, M., Wu, E.J. \& Yahalom, J. (2005). Intensity-modulated radiotherapy for lymphoma involving the mediastinum. Int J Radiat Oncol Biol Phys, 62: 198-206.

Sachs, R.K., Shuryak, I., Brenner, D., Fakir, H., Hlatky, L. \& Hahnfeldt P. (2007). Second cancers after fractionated radiotherapy: stochastic population dynamicseffects. J Theor Biol, 249:518-531.

Constine, L.S., Tarbell, N. \& Hudson, M.M. et al. (2008). Subsequent malignancies in children treated for Hodgkin's disease: associations with gender and radiation dose. Int $\mathrm{J}$ Radiat Oncol Biol Phys, 72:24-33.

Weber, D.C., Johanson, S., Peguret, N., Cozzi, L. \& Olsen, D.R. (2011). Predicted risk of radiation-induced cancers after involved field and involved node radiotherapy with or without intensity modulation for early-stage hodgkin lymphoma in female patients. Int J Radiat Oncol Biol Phys, 81:490-497.

Akkas, E.A. \& Guvenc, O. (2020). Boyun ve mediasten tutulumlu hodgkin lenfoma olgularında Butterfly VMAT tekniği avantajlı midır?. Turk J Clin Lab, 4: 274-281.

Fiandra, C., Filippi, A.R. \& Catuzzo, P. et al. (2012). Different IMRT solutions vs. 3D-Conformal Radiotherapy in early stage Hodgkin's lympho $\neg$ ma: dosimetric comparison and clinical considerations. Radiati $\neg$ on Oncology, 7: 186.

Lloyd, S., Aurand, A. \& Wagner. H. (2014). Hodgkin Lymphoma With Bulky Mediastinal Disease: A Comparison of AP/PA, IMRT, and Volumetric Arc Plans. Int J Radiat Oncol Biol Phys, 90(1): 672. 